\title{
Introduction to the Emerging Topics in Electronic Government Minitrack
}

\author{
Elin Wihlborg \\ Department of Management, \\ Linköping University \\ Sweden \\ elin.wihlborg@liu.se
}

\author{
Theresa A. Pardo \\ Center for Technology in \\ Government \\ University at Albany/SUNY \\ United States \\ tpardo@ctg.albany.edu
}

\author{
Lei Zheng \\ School of International \\ Relations and Public Affairs \\ Fudan University \\ China \\ zhengl@,fudan.edu.cn
}

The e-Government Emerging Topics Minitrack provides a home for incubating new topics and trends in e-Government research. e-Government is still evolving as an academic field. As the selection of papers here indicates, this evolution is characterized by the continued creation and integration of approaches, methods and subfields. Even as the foundations of the field continue to be clarified and refined, this track provides a venue for work focused on the emerging topics, novel issues, and innovations in the use of technology in e-Government research. The 2018 offering of the E-Government Emerging Topics Minitrack presents four papers representing different conceptualizations indicating different emerging paths of e-government research.

In the first paper: "Providing Public Value through Data Sharing: Understanding Critical Factors of Food Traceability for Local Farms and Institutional Buyers", Mila Gasco-Hernandez, Wenhui Feng and J. Ramon Gil-Garcia address how large data sets outside government agencies can be used to contribute to implementation of policies and regulations. They present a case study of how small farms and institutional buyers can be incentivized to share their data in a way that contributes to food safety, public health, and other societal goals. The analyses show that initiatives that show the benefits of having a wholechain food traceability system, policies and regulations, and participation in training activities are key incentives in creating new data sharing capability.

The second paper of this minitrack, authored by Nadine Ogonek and Jörg Becker, is a comparative case study of the implementation of e-government in two federal states Australia and Germany. The overall research question is communicated by the title of the paper: "Can we Learn from Down Under How to Rise Up in E-Government? A Comparative Analysis of the Public Sector Competences in the German and Australian Higher Education Systems". The study is based on a content analysis of 126 higher education study programs with a link to the public sector in these states. The study shows that the differences regarding the delivered content and the respective competences may explain why Australia scores better in international ranking of e-government.

The third paper, "Factors Promoting the Collection of Performance Measurement: Evidence from US Local Governments" is written by Rashmi Krishnamurthy, Kevin Desouza, Gregory Dawson and Alfred Ho. The paper focuses on how and why performance measurement is used as a part of government reform efforts and how it is embedded into e-government structures. An analysis of local and regional agencies in the US shows through a cluster analysis that combinations of organizational and external factors influence the outcome.

The final paper of the Emerging Topics Minitrack, "The Saliency and Urgency of Enterprise Data Management in the Public Sector?" by Teresa Harrison, Theresa Pardo, Mila Gasco and Donna Canestraro, argues that enterprise data management is a key enabler for new and innovative uses of data. The authors illustrate the salience and urgency of enterprise data management through three vignettes that highlight the potential of such efforts to reorganize the public sector along new data oriented lines. A focus on the role of governance and the chief data officer as key enablers to creating public value from data highlight the need for research in these areas.

These papers all contribute to the ET Minitrack's goal by extending our understanding of the foundations of digital government. These papers show different outcomes in different contexts, both between states and policy sectors as well as on different levels of our multi-level government systems. The second paper also contributes to the goal of the track by providing a reflection of how, as our foundations emerge, can we best educate the next generation to ensure future generations can leverage innovations in policy, management, and technology in the interest of citizens and society. Taken together these papers show the palette of theories and methods used to contribute both on analytical and practical developments and trends to digitalization of governments around the world. 